\title{
Prevalência de quedas em idosos cadastrados em um Centro de Saúde de Belo Horizonte-MG
}

\author{
Prevalence of falls in older adults enrolled in a Health Center of Belo Horizonte-MG \\ Prevalencia de caídas en ancianos inscritos en un Centro de Salud de Belo Horizonte-MG

\section{Tânia Couto Machado Chianca', Caroline Rodrigues de Andrade', Juliana Albuquerque', Luísa Cristina Crespo Wenceslau', Luiza Ferreira Ribeiro Tadeu', Tamara Gonçalves Rezende Macieira', Flávia Falci Ercole'}

\author{
'Universidade Federal de Minas Gerais, Escola de Enfermagem, Departamento de Enfermagem Básica. \\ Belo Horizonte-MG, Brasil.
}

Submissão: 05-07-2011 Aprovação: 15-04-2013

\begin{abstract}
RESUMO
A queda é utilizada como indicador de qualidade em serviços especializados no cuidado de idosos, sendo também considerado importante indicador de qualidade de vida de idosos em todos os ambientes. Realizou-se um estudo de corte transversal de 108 idosos cadastrados no Programa de Saúde da Família, em um Centro de Saúde de Belo Horizonte, Minas Gerais, com o objetivo de determinar a ocorrência de quedas nessa clientela, associando-as a algumas variáveis. Os dados foram coletados em prontuários e em visitas domiciliares por meio de entrevista estruturada, com instrumento específico. Observou-se que $64(59,3 \%)$ dos pacientes idosos já haviam sofrido quedas, havendo relação estatisticamente significativa $(p=0,01)$ entre capacidade cognitiva dos idosos e ocorrência das quedas. Recomenda-se o cuidado de enfermagem na identificação precoce dos idosos com maior chance de sofrerem quedas, especialmente daqueles que, além do risco de queda, apresentam risco aumentado de sofrer lesões graves decorrentes da mesma.
\end{abstract}

Descritores: Enfermagem; Acidentes por Quedas; Idoso, Diagnóstico de Enfermagem.

ABSTRACT
Fall is used as an indicator of quality in healthcare institutions specialized in elderly care and is also considered an important indicator of quality of life of seniors in all environments. This is a cross-sectional study with a convenience sample of 108 elderlies enrolled at a Health Center, in Belo Horizonte, Minas Gerais, in order to determine the occurrence of falls in elderly people and also look for its association with some variables. Data were collected from clinical records and interviews during home healthcare visits using a structured and specific instrument. It was found that $64(59.3 \%)$ of the elderly patients have already suffered falls, and a significant statistical relationship $(p=0.01)$ between the elderly cognitive ability and the occurrence of the falls. It is recommended that nursing care focuses in the early identification of the elderlies that are more likely to suffer falls, especially those who, in addition to the risk of falling are at increased risk of suffering serious injury originated from the falls.

Key words: Nursing; Accidental Falls; Elderly; Nursing Diagnoses.

\section{RESUMEN}

La caída se utiliza como indicador de la calidad de los servicios especializados en el cuidado de los ancianos, y también se considera un indicador importante de la calidad de vida de las personas mayores en todos los ambientes. Se realizó un estudio transversal con 108 ancianos inscritos en el Programa Salud de la Familia de un Centro de Salud, en Belo Horizonte, Minas Gerais, con el fin de determinar la incidencia de caídas en esta clientela, vinculándolas a algunas variables. Los datos se obtuvieron de los registros médicos y visitas a domicilio mediante entrevista estructurada, con uso de un instrumento específico. Se observó que 64 (59,3\%) de los ancianos ya había sufrido caídas, y una relación estadísticamente significativa (p $=0,01$ ) entre la capacidad cognitiva de los ancianos y las caídas. Se recomienda la identificación temprana de las personas mayores más propensas a sufrir caídas, especialmente aquellas que, además del riesgo de caídas, tienen un mayor riesgo de sufrir lesiones graves derivados de las mismas.

Palabras clave: Enfermería; Las Caídas Accidentales; Ancianos; Diagnóstico de Enfermería. 


\section{INTRODUÇÃO}

O perfil demográfico mundial tem sofrido uma transição caracterizada pelo aumento expressivo da população idosa, tanto em países desenvolvidos como em desenvolvimento(1). Tal fato se deve aos avanços nas áreas de saúde, tecnologia e indústria farmacêutica, além da adoção de hábitos de vida saudáveis que prestigiem a prática de exercícios físicos, a manutenção de uma dieta balanceada e a eliminação ou diminuição do tabaco e álcool. A adoção de políticas públicas de saúde com o objetivo de promover a saúde e prevenir as doenças também têm contribuído para esse cenário.

Estima-se que em 2025 a população idosa mundial será de 1,2 bilhões e, em 2050, 2 bilhões(2). No Brasil, as projeções indicam aumento de 2 a $4 \%$ da população idosa a cada ano(1). Tais valores alertam para as conseqüências dessa transição demográfica, não apenas no âmbito da previdência social, como também do sistema de saúde. Assim, observa-se a necessidade de elaboração de planos de ação capazes de minimizar os efeitos do envelhecimento populacional numa população ainda não preparada para uma distribuição demográfica com grande prevalência de idosos.

Além dessa evidente transição demográfica, o aumento da população idosa acarreta também uma transição epidemiológica. As doenças infectocontagiosas, que eram responsáveis pelos elevados valores de morbimortalidade em décadas anteriores, têm sido substituídas pelas doenças e agravos não transmissíveis ${ }^{(3)}$. Se, por um lado, o aumento da população idosa demonstra melhoria nos fatores relacionados à longevidade, estudos populacionais realizados no país têm demonstrado que $85 \%$ dos idosos apresentam pelo menos um agravo ${ }^{(4)}$. Em geral as doenças nos idosos são crônicas e múltiplas exigindo acompanhamento de equipes multidisciplinares e internações freqüentes. As principais doenças são as referentes ao aparelho circulatório, respiratório, digestivo, neoplasias e aparelho geniturinário ${ }^{(5)}$. Essas patologias muitas vezes deixam seqüelas, prejudicando ou impossibilitando a realização das atividades da vida diária (AVDs) pelos idosos, que podem tornar-se dependentes, tendo assim sua qualidade de vida prejudicada.

As quedas constituem também importante fator relacionado à diminuição na qualidade de vida dos idosos, devido ao fato de ser a maior causadora de deficiência e até mesmo de morte ${ }^{(6)}$.

Uma definição encontrada na literatura para a causa das quedas afirma que "a queda se dá em decorrência da perda total do equilíbrio postural, podendo estar relacionada à insuficiência súbita dos mecanismos neurais e osteoarticulares envolvidos na manutenção da postura"(7). Alguns autores referem-se à queda como uma síndrome geriátrica por ser considerado um evento multifatorial e heterogêneo ${ }^{(8-9)}$.

Pessoas pertencentes a qualquer faixa etária apresentam risco de sofrer quedas. Entretanto, na população idosa, o risco de queda possui um grande significado, pois ocorre com grande freqüência e pode gerar incapacidade, injúria e morte.

Em 2000, a NANDA I ${ }^{(10)}$ incluiu em sua classificação de diagnósticos de enfermagem o diagnóstico de Risco de Quedas, definido como a "suscetibilidade aumentada para quedas que podem causar dano físico". Os fatores de risco apontados encontram-se subdivididos nas seguintes categorias: medicamentos, fisiológicos, cognitivos e ambientais, tanto em adultos como em crianças. A idade do indivíduo acima de 65 anos é um fator de risco de quedas ${ }^{(10)}$.

Os fatores de risco para a ocorrência de quedas podem ser divididos em intrínsecos e extrínsecos. Os fatores intrínsecos são aqueles relacionados às características da pessoa e às mudanças associadas à idade. Dentre eles, os mais comuns são fraqueza muscular e modificações na marcha. Estas ocorrem devido a alterações no sistema músculo-esquelético e nos sistemas nervoso central e periférico, acarretando modificações na velocidade angular pélvica e na força de impulsão dos pés. Também são considerados fatores intrínsecos de queda as deficiências ocular, auditiva e cognitiva, o uso de medicamentos, principalmente hipotensores, sedativos e hipnóticos, idade avançada, mobilidade prejudicada e, histórico anterior de quedas ${ }^{(6,11)}$.

Os fatores extrínsecos são geralmente relacionados a ambientes que oferecem perigo ao idoso. Estão incluídos entre eles a presença de piso escorregadio, tapetes soltos, iluminação inadequada, presença de entulhos, escadas, armários e interruptores fora do alcance, além do uso de calçados inadequados e pobre distinção de cores entre paredes e mobília. Este último está relacionado ao fato de que somada aos problemas de acuidade visual, a homogeneidade de cores no ambiente dificulta a orientação espacial do idoso, podendo acarretar quedas ${ }^{(6,11)}$. Esses estudos utilizaram a revisão narrativa de literatura e levantamento com análise descritiva de dados.

A população idosa é vulnerável às ocorrências de quedas e estas causam prejuízos aos idosos, contribuem para a diminuição na qualidade de vida e geram gastos aos cofres públicos à medida que demandam maior utilização dos serviços de saúde. Por isto é importante determinar a prevalência de quedas entre os idosos, na população em geral e mais especificamente em uma população atendida em um Centro de Saúde de Belo Horizonte, Minas Gerais e associá-la às variáveis apontadas na literatura.

\section{MÉTODOS}

Trata-se de um estudo cross-seccional realizado durante os meses de agosto a dezembro de 2008, conduzido em áreas de abrangência do Centro de Saúde Heliópolis, localizado na cidade de Belo Horizonte, Minas Gerais, e pertencentes ao Programa de Saúde da Família (PSF). A metodologia empregada seguiu os seguintes passos: seleção dos idosos a partir do cadastro realizado pelos agentes comunitários de saúde; coleta de dados no domicilio dos idosos realizado por estudantes do Curso de Enfermagem da Universidade Federal de Minas Gerais, através de instrumento contendo os fatores de risco intrínsecos e extrínsecos de quedas ${ }^{(11)}$.

Os dados dos pacientes foram coletados em prontuários e durante a realização de visitas domiciliares mediante roteiro de entrevista estruturada. O instrumento foi composto por informações demográficas, sociais e clínicas que contemplavam elementos relativos aos fatores de risco intrínsecos e extrínsecos relacionados a quedas entre idosos. Entre os fatores intrínsecos foram considerados: sexo, idade, escolaridade, 
com quem mora, grau de dependência, diagnóstico médico, medicamentos que utiliza, sinais e sintomas que predispõem à queda, estado mental, estado emocional, grau de dependência, equilíbrio, marcha e ocorrência de quedas. Entre os fatores extrínsecos para quedas foram incluídos: presença de piso escorregadio, tapetes soltos, iluminação inadequada, entulhos, escadas, armários e interruptores fora do alcance e uso de calçados inadequados ${ }^{(6,10-11,13)}$.

Ainda foram coletados informações relacionadas ao "Miniexame do estado mental" (MEEM) de Folstein adaptado para a população brasileira ${ }^{(12)}$ para a avaliação da capacidade cognitiva dos idosos.

Acuidade visual é comumente mensurada pela tabela de Snellen. É uma avaliação de percepção de forma e diferenciação entre pequenos detalhes acerca dos objetos. O método prevê a leitura com base em letras " $E$ ", posicionadas em fileira e voltadas nas quatro direções em um cartaz que foi colocado a 6 metros de distância do idoso. O idoso era solicitado a ler com cada olho separadamente e com ambos, medindo-se a capacidade de ler as letras em diferentes tamanhos e determinando-se a acuidade visual. Os dois números obtidos representam o quanto da distância do cartaz o idoso foi capaz de ler com sucesso as letras e de determinar sua direção. A pontuação 6/6 significa uma acuidade visual preservada; moderada é aquela onde o nível de visão está prejudicada (até $6 / 24$ ), baixa (má acuidade visual com campo visual entre 3/60 e 6/60) e com déficit visual severo (campo de visão severamente reduzido, de 6/60 ou acima) $)^{(14)}$.

A população de idosos cadastrados no PSF era de 499 homens e 732 mulheres, totalizando 1.231 idosos, por ocasião da coleta de dados. A amostra de conveniência foi composta por 108 indivíduos para os quais foi realizado visita domiciliar em acompanhamento de Agentes Comunitários de Saúde (ACS). Foram determinados os seguintes critérios de elegibilidade para inclusão na amostra: ter 60 anos ou mais de idade; ser residente na cidade; ser atendido no Centro de Saúde; consentir em participar da pesquisa ou ter sua participação autorizada por responsável, a partir da assinatura de um Termo de Consentimento Livre e Esclarecido.

O Programa Statistic Package for Social Sciences - SPSS (15.0) foi utilizado para entrada, elaboração do banco de dados e análise. Foi procedida análise estatística descritiva, com distribuição de frequências simples e acumuladas, medidas de tendência central como média e mediana e medidas de variabilidade como o desvio-padrão. Para verificação de associação entre fatores de risco e queda utilizou-se o teste de proporção Qui-quadrado de Mantel-Haenszel (variáveis categóricas) e teste t-Student (variáveis contínuas), Intervalo de Confiança (IC 95\%) e Nível de Significância Estatística de $p \leq 0,05$. A prevalência foi estabelecida considerando o número de idosos (casos antigos e novos) que sofreram quedas durante um período de cinco meses.

O estudo foi aprovado pelo Comitê de Ética e Pesquisa da Universidade Federal de Minas Gerais, Parecer ETIC 681/08, e seguiu o estabelecido na normatização 196/96 referente aos aspectos éticos observados quando da realização de pesquisas realizadas em seres humanos.

\section{RESULTADOS}

\section{Características psicossociais e demográficas}

A amostra foi constituída por 108 idosos, dentre os quais 73 $(67,6 \%)$ eram do sexo feminino e $35(32,4 \%)$ do sexo masculino. A média de idade foi de 75,91 anos e desvio-padrão de 7,34, idade mínima de 61 anos e máxima de 95 anos. O perfil sociodemográfico da amostra está apresentado na Tabela 1.

Tabela 1 - Perfil sociodemográfico de amostra de idosos usuários de um PSF. Belo Horizonte, MG, 2008.

\begin{tabular}{llcc}
\hline Caracterização dos idosos $(\mathrm{n}=108)$ & $\mathbf{n}$ & $\%$ \\
\hline Sexo & Masculino & 35 & 32,4 \\
& Feminino & 73 & 67,6 \\
Raça & Branco & 55 & 50,9 \\
& Pardo & 30 & 27,8 \\
& Negro & 23 & 21,3 \\
Estado civil & Casado & 11 & 10,2 \\
& Viúvo & 97 & 89,8 \\
Grau de escolaridade & Analfabeto & 16 & 14,8 \\
& Ensino fundamental incompleto & 77 & 71,3 \\
& Ensino fundamental completo & 15 & 13,9 \\
Tipo de moradia & Própria & 98 & 90,7 \\
& Alugada & 4 & 3,7 \\
& Cedida & 6 & 5,6 \\
Reside & Sozinho & 1 & 0,9 \\
& Com 1 pessoa & 14 & 13,0 \\
& Com 2 pessoas & 32 & 29,6 \\
& & 56,4 \\
\hline
\end{tabular}

Dentre os 108 idosos estudados, 93 idosos (86,1\%) informaram receber menos de 03 salários mínimos de renda mensal familiar e todos utilizam o Sistema Único de Saúde (SUS) como principal meio para se obter serviços de atendimento de saúde.

Com relação à moradia, 36 idosos (33,3\%) apresentaram residência com 06 cômodos; seguidos por 26 (24,1\%) e 25 $(23,1 \%)$, com 07 e 05 cômodos, respectivamente. Apenas 01 idoso $(0,9 \%)$ informou morar sozinho e $61(56,4 \%)$ residem com mais de 03 moradores no domicílio.

\section{Características psicobiológicas}

Com relação ao Índice de Massa Corporal (IMC), 52 $(48,1 \%)$ idosos foram classificados como possuindo sobrepeso; $38(35,2 \%)$ na faixa normal de peso; $13(12,0 \%)$ foram classificados como obesos de classe I e $04(3,7 \%)$ como sendo baixo peso e 82 idosos (75,9\%) afirmaram não realizar nenhum tipo de atividade física. 
Quanto à acuidade visual $85(78,7 \%)$ idosos foram avaliados como possuindo déficit visual moderado; 17 (15,7\%), déficit visual severo; e 06 (5,6\%) como possuindo a acuidade visual preservada. Por sua vez, $60(55,6 \%)$ idosos informaram utilizar óculos como dispositivo de auxílio à visão e 12 $(11,1 \%)$ de usar dispositivo de auxílio à audição.

Com relação à mobilidade física, apenas 08 (7,4\%) idosos faziam uso de dispositivos de auxílio à locomoção, sendo que $04(3,7 \%)$ utilizavam cadeiras de rodas, $03(2,8 \%)$, bengalas ou muletas e $01(0,9 \%)$, andador. Também foi avaliada a adequação do calçado utilizado e verificou-se que 24 (22,2\%) idosos não utilizavam calçados adequados à idade.

Tabela 2 - Relação entre a classificação pelo Miniexame do estado mental e a ocorrência de quedas de idosos usuários ( $n=108)$ de um PSF. Belo Horizonte, MG, 2008.

\begin{tabular}{|c|c|c|c|c|c|c|}
\hline Qu & \multicolumn{2}{|c|}{ Sim } & \multicolumn{2}{|c|}{ Não } & \multicolumn{2}{|c|}{ Total } \\
\hline pelo Mini-Mental & $\mathrm{n}$ & $\%$ & $\mathrm{n}$ & $\%$ & $N$ & $\%$ \\
\hline Apresenta algum problema cognitivo & 23 & 21,3 & 48 & 44,4 & 71 & 65,7 \\
\hline Não apresenta problema cognitivo & 21 & 19,4 & 16 & 14,8 & 37 & 34,3 \\
\hline Total & 44 & 40,7 & 64 & 59,2 & 108 & 100 \\
\hline
\end{tabular}

\section{Características da moradia}

Avaliou-se o projeto arquitetônico das residências, buscando fatores de risco para a ocorrência de quedas ${ }^{(10)}$. Foram encontradas inadequações domésticas que poderiam favorecer as quedas, como piso, iluminação, organização e altura do mobiliário dos cômodos. Quanto à presença de obstáculos, $86(79,6 \%)$ residências possuíam tapetes, sendo que em 78 $(72,2 \%)$, estes se apresentavam como empecilho à movimentação dos idosos nos ambientes. A altura do vaso sanitário também foi avaliada, e em $62(57,4 \%)$ residências estes foram considerados compatíveis com a altura do idoso (Tabela 3).

\section{Utilização de medicamentos}

Entre os idosos, $62(57,4 \%)$ referiram fazer uso de medicamentos diariamente. Estes são na sua maioria para o tratamento da hipertensão arterial e diabetes mellitus. Entretanto a referência de uso diário de medicamentos como antiparkinsonianos, antidepressivos, hipoglicemiantes, antiepiléticos, antipsicóticos é baixa entre eles.

\section{Ocorrência de quedas}

Encontrou-se uma proporção de idosos que apresentaram quedas no último ano de 59,3\% (64/108). Destes, 41 (64,1\%) referiram ter sofrido mais de um evento queda no último ano. Identificou-se que $48,44 \%$ dos idosos sofreram queda da própria altura (31/64). Entre os locais de ocorrência de quedas no presente estudo o evento foi mais freqüente nos ambientes do quintal e quarto $(12,5 \%$ e $6,3 \%$, respectivamente). Entre os idosos, 86 (79,6\%) afirmaram insegurança ou medo de cair.

Tabela 3 - Relação entre projeto arquitetônico das casas e histórico de quedas em idosos usuários $(n=108)$ de um PSF de Belo Horizonte, MG, 2008.

\begin{tabular}{|c|c|c|c|c|c|c|}
\hline \multirow{3}{*}{ Variáveis } & \multicolumn{4}{|c|}{ Adequado } & \multirow{3}{*}{ OR (IC 95\%) } & \multirow{3}{*}{ Valor $p$} \\
\hline & \multicolumn{2}{|c|}{ Sim } & \multicolumn{2}{|c|}{ Não } & & \\
\hline & Freq. & $\%$ & Freq. & $\%$ & & \\
\hline *Piso da sala & 42 & 38,9 & 65 & 60,2 & $1,23[0,55-2,72]$ & 0,61 \\
\hline Piso da cozinha & 31 & 28,7 & 77 & 71,3 & $0,93[0,40-2,22]$ & 0,87 \\
\hline Piso do banheiro & 17 & 15,7 & 91 & 84,3 & $1,31[0,45-4,14]$ & 0,62 \\
\hline Piso do quarto & 31 & 28,7 & 77 & 71,3 & $0,93[0,40-2,22]$ & 0,87 \\
\hline Piso do corredor & 31 & 28,7 & 77 & 71,3 & $0,93[0,40-2,22]$ & 0,87 \\
\hline *Iluminação da sala & 32 & 29,6 & 75 & 69,4 & $1,03[0,44-2,44]$ & 0,95 \\
\hline Iluminação da cozinha & 33 & 30,6 & 75 & 69,4 & $1,08[0,47-2,55]$ & 0,85 \\
\hline Iluminação do banheiro & 18 & 16,7 & 90 & 83,3 & $1,46[0,50-4,55]$ & 0,49 \\
\hline Iluminação do quarto & 33 & 30,6 & 75 & 69,4 & $1,08[0,47-2,55]$ & 0,85 \\
\hline Iluminação do corredor & 32 & 29,6 & 76 & 70,4 & $1,01[0,43-2,38]$ & 0,99 \\
\hline *Organização da mobília da sala & 32 & 29,6 & 75 & 69,4 & $0,86[0,37-2,02]$ & 0,72 \\
\hline Organização da mobília da cozinha & 31 & 28,7 & 77 & 71,3 & $0,93[0,40-2,22]$ & 0,87 \\
\hline Organização da mobília do banheiro & 17 & 15,7 & 91 & 84,3 & $1,31[0,45-4,14]$ & 0,62 \\
\hline Organização da mobília do quarto & 42 & 38,9 & 66 & 61,1 & $1,02[0,46-2,27]$ & 0,96 \\
\hline *Altura da mobília da sala & 43 & 39,8 & 64 & 59,3 & $1,16[0,51-2,48]$ & 0,78 \\
\hline Altura da mobília da cozinha & 17 & 15,7 & 91 & 84,3 & $1,31[0,45-4,14]$ & 0,62 \\
\hline Altura da mobília do banheiro & 17 & 15,7 & 91 & 84,3 & $1,31[0,45-4,14]$ & 0,62 \\
\hline Altura da mobília do quarto & 26 & 24,1 & 82 & 75,9 & $1,13[0,46-2,89]$ & 0,77 \\
\hline
\end{tabular}

Nota: *Variáveis com perda de informação. 


\section{Ocorrência de quedas e associação com as variáveis} independentes

Observou-se uma relação estatisticamente significativa entre a ocorrência de quedas e a capacidade cognitiva dos idosos $(p=0,004)$.

Assinala-se que não foi encontrada associação estatisticamente significativa entre a ocorrência de quedas e acuidade visual $(p=1)$ e nem com outras variáveis como o uso de várias classes de medicamentos; insegurança ou medo de sofrer queda $(p=0,98)$, uso de calçados inadequados $(p=0,40)$, presença de tapetes no domicilio $(p=0,15)$, iluminação inadequada em vários ambientes da residência, presença de escada na casa $(p=0,65)$.

Esses idosos apresentaram homogeneidade para os aspectos sociodemográficos como raça, estado civil, grau de escolaridade e tipo de moradia, não sendo encontradas associação estatisticamente significativa entre essas variáveis e a ocorrência de quedas.

\section{DISCUSSÃO}

Para o desempenho eficiente das tarefas de vida cotidiana é essencial que o ser humano mantenha o equilíbrio, sendo que este pode ser afetado tanto pelo processo de envelhecimento como pelas doenças crônicas( ${ }^{(15)}$. A ocorrência de queda em idosos está estreitamente ligada a distúrbios no equilíbrio. Estudo transversal para avaliar e comparar o equilíbrio funcional de idosos da comunidade sem história de quedas, com uma queda e com quedas recorrentes observou que idosos com histórico de ocorrência de quedas apresentaram comprometimento na função de equilíbrio em relação àqueles sem quedas ${ }^{(15)}$. No presente estudo foi encontrada uma proporção de quedas entre os idosos de $59,3 \%(64 / 108)$.

Essa é muito elevada considerando resultados de outras pesquisas como um estudo transversal com 180 idosos residentes em asilos no Rio Grande do Sul onde encontrou-se uma prevalência de quedas de $38,3 \%$. As quedas ocorreram mais no ambiente do quarto dos idosos, em idosos separados e divorciados, com depressão e que faziam uso de maior quantidade de medicamentos de uso contínuo ${ }^{(16)}$.

Estudo de coorte de 1.667 idosos de 65 anos ou mais residentes na comunidade de São Paulo, com seguimento de dois anos, teve como objetivo identificar fatores associados às quedas e àquelas recorrentes. Cerca de $31 \%$ dos idosos apresentaram história de queda e cerca de $11 \%$ afirmaram ter sofrido duas ou mais quedas. Durante o seguimento, 53,4\% dos idosos não referiram quedas, $32,7 \%$ afirmaram ter sofrido queda em pelo menos um dos inquéritos e 13,9\% relataram quedas em ambos os inquéritos. O modelo preditivo de quedas recorrentes foi composto das variáveis: ausência de cônjuge, não ter o hábito de ler, história de fratura, dificuldade em uma a três atividades de vida diária, dificuldade em quatro ou mais atividades de vida diária e entre aqueles idosos com visão comprometida ${ }^{(13)}$.

A variável avaliação da capacidade cognitiva foi considerada fator de risco para as quedas em idosos, apresentando associação estatisticamente significativa. Este achado também foi apontado em estudo transversal para avaliar e comparar o equilíbrio funcional de idosos da comunidade sem história de quedas, com uma queda e com quedas recorrentes ${ }^{(15)}$. Neste foi demonstrado que a capacidade cognitiva é um fator de interferência na avaliação do equilíbrio funcional dos idosos. A diminuição na capacidade cognitiva está relacionada ao enveIhecimento fisiológico do indivíduo, podendo estar associada a diversos fatores, dentre eles a diminuição no número de neurônios e atrofia cerebral com dilatação de sulcos e ventrículos $^{(17)}$. Estas alterações cerebrais podem resultar em quadros de demência, com níveis variados de gravidade.

Interessante observar o elevado número de idosos $(79,6 \%)$ que referiram possuir receio de sofrer quedas (86/108). O medo de cair pode prejudicar a autoconfiança e segurança emocional do idoso, podendo refletir negativamente na realização das atividades de vida diária (AVDs) e nas atividades instrumentais de vida diária (AIVDs). Em estudo realizado em São Paulo, encontrou-se que existe associação entre história de ocorrência de quedas e a dificuldade em executar AVDs e AIVDs ${ }^{(13)}$.

Em estudo de levantamento de dados com análise descritiva ${ }^{(18)}$, encontrou-se que o medo de sofrer novas quedas foi a segunda maior conseqüência apontada pelos idosos que haviam caído e já foi demonstrado que existe associação $(p=0,001)$ entre o medo de cair e a ocorrência de quedas (19). No presente estudo, entretanto, não foi encontrado uma relação estatisticamente significativa entre o medo de cair e a ocorrência de quedas $(p=0,98)$. Sabe-se que o medo de cair pode levar os idosos a sofrer quedas devido a muitas vezes ao fato de terem perdido a independência, a competência para a realização de suas atividades de vida diária, sedentarismo e restrição de atividade e sedentarismo. Estudo transversal randomizado estabeleceu a prevalência do medo de cair em uma população de idosos com mobilidade, equilíbrio dinâmico, risco e histórico de quedas ${ }^{(20)}$.

As frequências encontradas no presente estudo nas variáveis sócio-demográficas, psicobiológicas e ambiente são corroboradas em estudo descritivo realizado para identificar os fatores relacionados ao diagnóstico de enfermagem "risco de quedas" entre 51 idosos residentes em uma unidade básica de saúde paulista. Neste encontrou-se que todos os idosos apresentavam o problema risco para quedas e que este estava relacionado a inúmeros fatores intrínsecos e extrínsecos. Os fatores relacionados ao risco de quedas mais encontrados referiram-se ao sexo feminino (34-66,6\%), acuidade visual diminuída (24-47\%), história de quedas (26-50,98\%), presença de piso escorregadio $(23-45,08 \%)$ nos domicílios, banheiro sem piso antiderrapante (31-60,76\%), tapetes e objetos soltos (25$49 \%$ ) nos ambientes, baixa luminosidade $(12-23,52 \%)$, uso de calçado inadequado (38-74,48\%). Além disso, encontrou-se alterações funcionais nos idosos como dificuldade de movimentar braços e pernas (10-19,60\%) e falta de firmeza nas pernas $(5-9,80 \%)$, hipertensão arterial $(27-52,92 \%)$ e diabetes mellitus $(14-27,44 \%)$, além de referência a problemas como distúrbio do sono (13-25,48\%) e vertigem $(7-13,72 \%)^{(21)}$.

Outra variável testada foi acuidade visual, não se encontrando relação estatisticamente significativa em relação à história de ocorrência de quedas entre os idosos. Neste estudo 
encontrou-se que 102 idosos (94,4\%) apresentavam déficit visual moderado a severo e $60(55,6 \%)$ usavam dispositivo para correção visual. Na literatura, encontra-se que diminuição da acuidade visual é fator de risco para quedas em idosos ${ }^{(9,}$ 22). No presente estudo apenas 06 (5,6\%) idosos possuíam visão preservada e $96(88,9 \%)$ apresentava audição preservada. Com a idade ocorre diminuição na percepção auditiva, visual e sensorial e essa contribui para a perda na sensação de equilíbrio que pode levar a um aumento no risco de sofrer quedas $^{(7-8)}$.

No presente estudo não foi encontrada relação estatisticamente significativa entre as condições do piso, iluminação dos cômodos, presença de escadas, altura e organização do mobiliário com a ocorrência de quedas. Estudo desenvolvido por Marin e colaboradore ${ }^{(11)}$ contraria os resultados encontrados neste trabalho.

Não foi encontrada significância estatística entre a utilização de medicamentos e a ocorrência de quedas. Estudo desenvolvido $^{(16)}$ corrobora que medicamentos como antipsicóticos, hipnóticos e sedativos, antiepiléticos, hipoglicemiantes, antidepressivos e antiparkinsonianos são usados com frequência por idosos, com relação estatisticamente significativa entre ocorrência de quedas e uso de psicotrópicos por idosos. Neste foi observado que $28,1 \%$ dos idosos que relataram quedas recorrentes estavam em uso de alguma das medicações pesquisadas, sinalizando para o fato de que estes indivíduos poderiam estar sujeitos aos efeitos colaterais destes medicamentos e tendo o risco para sofrer quedas aumentado ${ }^{(15)}$. Entretanto, não se encontrou no presente estudo relação estatisticamente significativa entre o uso desses medicamentos e a história de ocorrência de quedas.

Parece que a falta de relação entre quedas e as diversas variáveis normalmente encontradas na literatura e associadas ao problema constitui uma particularidade desta amostra de conveniência de idosos em especial. Alude-se este fato ao pequeno número de idosos, a pertenceram a uma unidade básica de saúde onde equipe de saúde da família é atuante, com participação de estudantes de graduação da área de saúde que desenvolve atividade de cuidado corriqueira de visita domiciliar, educação para a saúde e orientações relativas a tratamento, ambiente, prevenção e reabilitação.

\section{LIMITAÇÕES}

Em estudos de prevalência deste tipo, podem ocorrer limitações relacionadas ao viés de memória em que a confiabilidade dos dados coletados pode estar comprometida, uma vez que esta depende da lembrança, da memória do indivíduo. Neste estudo, a lembrança, algumas vezes, pode ter estado prejudicada devido ao déficit cognitivo apresentado pelos idosos.

Outro viés importante, relacionado aos estudos de prevalência, é o problema da sobrevivência. Os pacientes incluídos no estudo são casos prevalentes. Aqueles indivíduos que se curam ou que morrem mais rapidamente devido ao evento estudado (quedas) têm menos chances de serem incluídos no estudo.

\section{CONCLUSÃO E IMPLICAÇÕES PARA A PRÁTICA GERONTOLÓGICA}

No presente estudo, o fator de risco capacidade cognitiva dos idosos apresentou-se relacionada estatisticamente com a ocorrência de quedas. Entretanto, algumas variáveis analisadas como a acuidade auditiva e visual, insegurança ou medo de cair, características do ambiente, uso de medicamentos e outras no estudo não apresentaram associação com o risco de quedas nos idosos.

Acredita-se que a acuidade visual não foi um fator relevante para o risco de quedas devido ao fato de que apenas uma pequena parcela da amostra $(15,7 \%)$ apresentou déficit visual severo, além de mais da metade $(55,6 \%)$ dos idosos utilizarem dispositivos de auxílio à visão, o que reduz a possibilidade de quedas. Quanto às variáveis relacionadas ao uso de medicamentos antipsicóticos, antiepiléticos, antidepressivos, ansiolíticos, opiáceos, antiparkinsonianos não foram significativas para a ocorrência das quedas talvez pelo fato de apenas uma pequena parcela da amostra fazer uso dessas classes de medicamentos. Estudos mais abrangentes devem ser conduzidos para testar a relação dessas variáveis e a ocorrência de quedas.

A queda é um evento freqüente e limitante, sendo considerado um marcador de fragilidade, institucionalização, de declínio na saúde e morte em idosos. O risco de quedas aumenta significativamente com o avançar da idade, sendo um grande problema de saúde pública devido ao aumento expressivo no número de idosos na população e à maior longevidade das pessoas, aumentando a demanda por cuidados de longa duração.

Diversos fatores de risco e múltiplas causas interagem como agentes determinantes e predisponentes para a ocorrência de quedas em idosos, tanto para quedas acidentais quanto para quedas recorrentes, impondo aos profissionais de saúde o grande desafio de identificar os possíveis fatores de risco modificáveis e tratar os fatores etiológicos e comorbidades presentes.

Reconhece-se que as intervenções mais eficazes baseiam-se na identificação precoce dos idosos com maior chance de sofrerem quedas e especialmente aqueles que, além do risco de queda, apresentem também um risco aumentado de sofrer lesões graves decorrentes da mesma.

Por sua vez, os idosos tendem a subnotificar as quedas e creditam à idade seus problemas de equilíbrio e marcha, fazendo com que estas dificuldades de mobilidade sejam pouco consideradas ao se elaborar um plano de cuidados. Dessa forma, até que uma queda com uma conseqüência grave ocorra não é atribuída a devida importância ao problema.

Assim, evitar o evento de queda é considerado hoje uma conduta de boa prática gerontológica, tanto em hospitais e instituições de longa permanência quanto no ambiente domiciliar. Ressalta-se que a queda tem sido um dos indicadores de qualidade em serviços especializados no cuidado de idosos, sendo também considerada como um importante indicador de qualidade de vida de idosos em todos os ambientes. 


\section{REFERÊNCIAS}

1. Nasri F. O envelhecimento populacional no Brasil. Einstein 2008;6(1):5-6.

2. World Health Organization. Envelhecimento ativo: uma política de saúde. Brasília: Organização Pan-Americana da Saúde; 2005.

3. Ministério da Saúde (Brasil). Portaria nº. 2.528, de 19 de outubro de 2006. Dispõe sobre a aprovação da Política Nacional de Saúde do Idoso [portaria na internet]. Diário Oficial da União 22 out 2011 [acesso em 18 abr 2013]. Disponível em: < http://portal.saude.gov.br/portal/arquivos/pdf $/ 2528 \% 20$ aprova $\% 20$ a $\% 20$ politica $\% 20$ nacional $\% 20$ de $\% 20$ saude $\% 20$ da $\% 20$ pessoa $\% 20$ idosa.pdf $>$.

4. Teixeira MB. Empoderamento de idosos em grupos direcionados à promoção da saúde. Rio de Janeiro. Dissertação [Mestrado em Saúde Pública] -Fundação Oswaldo Cruz, Escola Nacional de Saúde Pública; 2002.

5. Mathias TAF, Jorge MHPM. Hospitalização e mortalidade em idosos: um exercício de análise comparativa. Ciênc Cuid Saúde 2005; 4(1):25-36.

6. Wang SY, Wollin J. Falls among older people: identifying those at risk. Nurs Older People 2004; 15(10):14-20.

7. Guimarães RM, Cunha UGV. Sinais e Sintomas Associados à Marcha. In: Guimarães RM, Cunha UGV. Sinais e Sintomas em Geriatria. Rio de Janeiro: Atheneu; 2004. p. 249-252.

8. Duthie EH, Katz PR. Geriatria Prática. Rio de Janeiro: Revinter; 2002.

9. Jacob Filho W, Kikuchi EL. Geriatria e Gerontologia básicas. Rio de Janeiro: Elsevier; 2011.

10. NANDA International. Diagnósticos de enfermagem da NANDA: definições e classificação (2010-2012). Porto Alegre: Artmed; 2013.

11. Marin MJS, Castilho NC, Myazato JM, Ribeiro PC, Candido DV. Características dos riscos para quedas entre idosos de uma unidade de saúde da família. REME Rev Min Enferm 2007; 11(4):369-74.

12. Bertolucci PHF, Brucki SMD, Campacci SR, Juliano Y.
O mini-exame do estado mental em uma população geral: impacto da escolaridade. Arq Neuropsiquiatr 1994; 52(1):1-7.

13. Perracini MR, Ramos LR. Fatores associados a quedas em uma coorte de idosos residentes na comunidade. Rev Saúde Pública 2002;36(6):709-16.

14. Potter PA, Perry AG. Fundamentos de Enfermagem. Rio de Janeiro: Elsevier; 2005.

15. Gonçalves DFF, Ricci NA, Coimbra AMV. Equilíbrio funcional de idosos da comunidade: comparação em relação ao histórico de quedas. Rev Bras Fisioter 2009;13(4):316-23.

16. Gonçalves LG, Vieira ST, Siqueira FV, Hallal PC. Prevalência de quedas em idosos asilados do município de Rio Grande, RS. Rev Saúde Pública 2008;42(5):938-45.

17. Nordon DG, Guimarães RR, Kozonoe DY, Mancilha VS, Neto VSD. Perda cognitiva em idosos. Rev Fac Ciênc Méd Sorocaba 2009;11(3):5-8.

18. Fabricio SCC, Rodrigues RAP, Junior MLC. Causas e conseqüências de quedas de idosos atendidos em hospital público. Rev Saúde Pública 2004;38(1):93-9.

19. Dalbere K, Crombez G, Vanderstraeten G, Willems T, Cambier D. Fear related avoidance of activities, falls and physical frailty: a prospective community-based cohort study. Age Ageing 2004; 33(4):368-73.

20. Lopes KT, Costa DF, Santos LF, Castro DP, Bastone AC. Prevalência do medo de cair em uma população de idosos da comunidade e sua correlação com mobilidade, equilíbrio dinâmico, risco e histórico de quedas. Rev Bras Fisioter 2009;13(3):223-9.

21. Marin MJS, Amaral FS, Martins IB. Identificando os fatores relacionados ao diagnostico de enfermagem "risco de quedas" entre idosos. Rev Bras Enferm 2004;57(5):560-4.

22. La Grow SJ, Robertson MC, Campbell AJ, Clarke GA, Kerse NM. Reducing hazard related falls in people 75 years and older with significant visual impairment: how did a successful program work? Inj Prev 2006;12(5):296-301. 